\title{
Close-to-Convexity of Convolutions of Classes of Harmonic Functions
}

\author{
Raj Kumar Garg' and Jay M. Jahangiri $\mathbb{D}^{2}$ \\ ${ }^{1}$ DAV University, Jalandhar, Punjab 144012, India \\ ${ }^{2}$ Mathematical Sciences, Kent State University, Burton, OH 44021-9500, USA \\ Correspondence should be addressed to Jay M. Jahangiri; jjahangi@kent.edu
}

Received 4 February 2018; Accepted 25 March 2018; Published 2 May 2018

Academic Editor: Teodor Bulboaca

Copyright @ 2018 Raj Kumar Garg and Jay M. Jahangiri. This is an open access article distributed under the Creative Commons Attribution License, which permits unrestricted use, distribution, and reproduction in any medium, provided the original work is properly cited.

For $j=1,2$ and for positive integers $m$ and $n$, we consider classes of harmonic functions $f_{j}=h_{j}+\overline{g_{j}}$, where $g_{1}(z)=z^{n} h_{1}(z)$ and $g_{2}^{\prime}(z)=z^{n} h_{2}^{\prime}(z)$ or $g_{1}^{\prime}(z)=z^{n} h_{1}^{\prime}(z)$ and $g_{2}^{\prime}(z)=z^{m} h_{2}^{\prime}(z)$, and we prove that their convolution $f_{1} * f_{2}=h_{1} * h_{2}+\overline{g_{1} * g_{2}}$ is locally one-to-one, sense-preserving, and close-to-convex harmonic in $|z|<1$.

\section{Introduction}

Let $\mathscr{A}$ denote the class of functions that are analytic in the open unit disk $\mathbb{E}:=\{z:|z|<1\}$ and let $\mathscr{A}^{\prime}$ be the subclass of $\mathscr{A}$ consisting of functions $h$ with the normalization $h(0)=$ $h^{\prime}(0)-1=0$. Consider the family of complex-valued harmonic functions $f=u+i v$, where $u$ and $v$ are real harmonic in $\mathbb{E}$. Such functions can be expressed as $f=h+\bar{g}$, where $h \in \mathscr{A}$ and $g \in \mathscr{A}$. By Lewy's Theorem (see [1,2] or [3]), a necessary and sufficient condition for the harmonic function $f=h+\bar{g}$ to be locally one-to-one and sense-preserving in $\mathbb{E}$ is that its Jacobian $J_{f}=\left|h^{\prime}\right|^{2}-\left|g^{\prime}\right|^{2}$ should be positive or equivalently if and only if $h^{\prime} \neq 0$ in $\mathbb{E}$ and the second complex dilatation $\omega$ of $f$ satisfies $|\omega|=\left|g^{\prime} / h^{\prime}\right|<1$ in $\mathbb{E}$. In the sequel, without loss of generality, we consider those locally one-toone and sense-preserving harmonic functions $f=h+\bar{g}$ that are normalized by $f(0)=h(0)=0$ and $f_{z}(0)=1$ and have the representation

$$
f(z)=h(z)+\overline{g(z)}=z+\sum_{n=2}^{\infty} a_{n} z^{n}+\overline{\sum_{n=1}^{\infty} b_{n} z^{n}} ; \quad z \in \mathbb{E} .
$$

The Hadamard product or convolution of two power series $h_{1}(z)=\sum_{n=1}^{\infty} a_{n} z^{n}$ and $h_{2}(z)=\sum_{n=1}^{\infty} c_{n} z^{n}$ is given by $h_{1}(z) *$ $h_{2}(z)=\left(h_{1} * h_{2}\right)(z)=\sum_{n=1}^{\infty} a_{n} c_{n} z^{n}$. Similarly, the convolution of two harmonic functions $f_{1}=h_{1}+\overline{g_{1}}$ and $f_{2}=h_{2}+\overline{g_{2}}$ is given by $f_{1} * f_{2}=h_{1} * h_{2}+\overline{g_{1} * g_{2}}$.

A simply connected proper subdomain $\mathbb{D}$ of the complex domain $\mathbb{C}$ is said to be convex if the linear segment joining any two points of $\mathbb{D}$ lies entirely in $\mathbb{D}$ and is said to be close-toconvex if its complement in $\mathbb{C}$ is the union of closed half-lines with pairwise disjoint interiors. Consequently, a univalent analytic or harmonic function $f: \mathbb{E} \rightarrow \mathbb{C}$ is said to be convex or close-to-convex in $\mathbb{E}$ if $f(\mathbb{E})$ is convex or close-to-convex there. For $-1 / 2 \leq \alpha<1$, a function $h \in \mathscr{A}^{\prime}$ is said to be in the class $\mathscr{K}(\alpha)$ if $\operatorname{Re}\left[1+z h^{\prime \prime}(z) / h^{\prime}(z)\right]>\alpha, z \in \mathbb{E}$. It can easily be verified that $\mathscr{K}(\beta) \subset \mathscr{K}(\alpha)$ if $-1 / 2 \leq \alpha<\beta<1$. If $h \in(\alpha)$ for $0 \leq \alpha<1$ in $\mathbb{E}$, then $h$ is said to be convex of order $\alpha$ in $\mathbb{E}$ (e.g., see [3] or [4]). A function $h \in \mathscr{K}(0)$ is simply called a convex function in $\mathbb{E}$.

Ruscheweyh and Sheil-Small [5] proved that the Hadamard product or convolution of two analytic convex functions is also convex analytic and that the convolution of an analytic convex function and an analytic close-to-convex function is close-to-convex analytic in the unit disk $\mathbb{E}$. Ironically, these results can not be extended to the harmonic case, since the convolution of harmonic functions, unlike the analytic case, proved to be very challenging.

Recently, Ahuja and Jahangiri [6] proved the following theorem. 
Theorem 1. Let the functions $h_{1} \in \mathscr{A}^{\prime}$ and $h_{2} \in \mathscr{A}^{\prime}$ be in the class $\mathscr{K}(\alpha)$ in $\mathbb{E}$. If either $g_{1}=z h_{1}, g_{2}^{\prime}=z h_{2}^{\prime}, \alpha \geq-1 / 2$ or $g_{1}^{\prime}=z^{n} h_{1}^{\prime}, g_{2}^{\prime}=z^{n} h_{2}^{\prime}, \alpha \geq 0$, then $F=h_{1} * h_{2}+\overline{g_{1} * g_{2}}$ is locally one-to-one, sense-preserving, and close-to-convex harmonic in $\mathbb{E}$.

The following question is asked in [6].

Question 2. Is Theorem 1 true for $g_{1}=z^{n} h_{1}$ and $g_{2}^{\prime}=z^{n} h_{2}^{\prime}$ if $n>1$ ?

In Theorem 3, we address Question 2. Moreover, in Theorem 4, we allow variations in the powers of $z$ for the dilatations of harmonic functions. Also note that the techniques presented here to prove our theorems are different from those used in [6].

Theorem 3. Let the functions $h_{1} \in \mathscr{A}^{\prime}$ and $h_{2} \in \mathscr{A}^{\prime}$ be so that $h_{1} * h_{2}$ is convex in $\mathbb{E}$. Set $g_{1}(z)=z^{n} h_{1}(z)$ and $g_{2}^{\prime}(z)=z^{n} h_{2}^{\prime}(z)$, where $n \in \mathbb{N}:=\{1,2,3, \ldots\}$. Then the convolution function $F(z)=h_{1}(z) * h_{2}(z)+\overline{g_{1}(z) * g_{2}(z)}$ is locally one-to-one, sensepreserving, and close-to-convex harmonic in $\mathbb{E}$.

Theorem 4. Let the functions $h_{1} \in \mathscr{A}^{\prime}$ and $h_{2} \in \mathscr{A}^{\prime}$ be convex of order $1 / 2$ in $\mathbb{E}$. Set $g_{1}^{\prime}(z)=z^{n} h_{1}^{\prime}(z), n \in \mathbb{N}:=\{1,2,3, \ldots\}$, and $g_{2}^{\prime}(z)=z^{m} h_{2}^{\prime}(z), m \in \mathbb{N}$. Then the convolution function $F(z)=h_{1}(z) * h_{2}(z)+\overline{g_{1}(z) * g_{2}(z)}$ is locally one-to-one, sensepreserving, and close-to-convex harmonic in $\mathbb{E}$.

In the following example, we demonstrate a case of closeto-convexity of convolutions of two harmonic functions.

Example 5. Consider

$$
\begin{aligned}
& f_{1}(z)=h_{1}(z)+\overline{g_{1}(z)}=\frac{z}{1-z}+\frac{\bar{z}}{1-z}+\log (1-z), \\
& f_{2}(z)=h_{2}(z)+\overline{g_{2}(z)}=\arctan z+\frac{1}{2} \overline{\log \left(1+z^{2}\right)} .
\end{aligned}
$$

For $j=1,2$, it is easy to verify that $g_{j}^{\prime}(z)=z h_{j}^{\prime}(z)$ and

$$
\begin{aligned}
F(z)= & H(z)+\overline{G(z)}=f_{1}(z) * f_{2}(z) \\
= & h_{1}(z) * h_{2}(z)+\overline{g_{1}(z) * g_{2}(z)} \\
= & \arctan z \\
& \quad \overline{\frac{1}{2} \log \left(1+z^{2}\right)-\frac{1}{2} \int_{0}^{z} \frac{1}{t} \log \left(1+t^{2}\right)} d t \\
= & \arctan z+\frac{1}{2} \sum_{n=1}^{\infty}(-1)^{n-1} \frac{2 n-1}{2 n^{2}} \overline{z^{2 n}} .
\end{aligned}
$$

Also,

$$
\begin{aligned}
\left|\frac{G^{\prime}(z)}{H^{\prime}(z)}\right| & =\left|z-\frac{\left(1+z^{2}\right) \log \left(1+z^{2}\right)}{2 z}\right| \\
& =\frac{|z|}{2}\left|1-\frac{z^{2}}{2}+\frac{z^{4}}{6}-\frac{z^{6}}{12}+\frac{z^{8}}{20}+\cdots\right|
\end{aligned}
$$

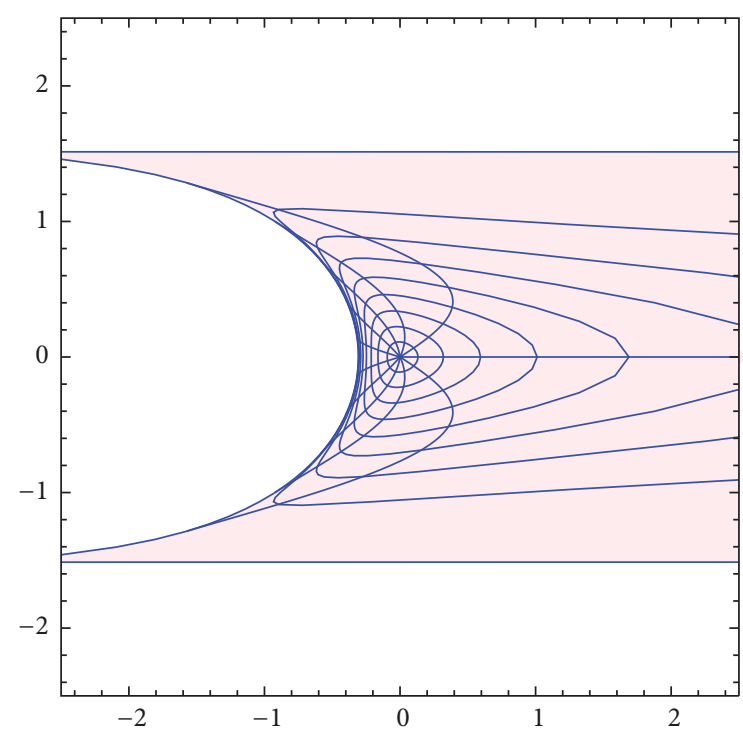

Figure 1: Image of $E$ under $f_{1}$.

$$
\begin{aligned}
& \leq \frac{|z|}{2}\left[1+\frac{|z|^{2}}{2}+\frac{|z|^{4}}{6}+\frac{|z|^{6}}{12}+\frac{|z|^{8}}{20}+\cdots\right] \\
& <\frac{1}{2}\left[1+\frac{1}{2}+\frac{1}{6}+\frac{1}{12}+\frac{1}{20}+\cdots\right]<\frac{1}{2}(2) \\
& =1 .
\end{aligned}
$$

Therefore, $f_{1} * f_{2}$ is locally one-to-one, sense-preserving, and close-to-convex harmonic in $\mathbb{E}$.

The images of $E$ under $f_{1}, f_{2}$, and $f_{1} * f_{2}$ are shown in Figures 1, 2, and 3, respectively.

\section{Preliminary Lemmas and Proofs}

To prove our theorems, we shall need the following four lemmas. Lemmas 6 and 9 are according to Clunie and SheilSmall [2], Lemma 7 is a well-established result by Robinson [7], and Lemma 8 is a celebrated result by Ruscheweyh and Sheil-Small [5].

Lemma 6. Let $g$ and $h$ be analytic in $\mathbb{E}$ so that $\left|g^{\prime}(0)\right|<$ $\left|h^{\prime}(0)\right|$. If $h+\epsilon g$ is close-to-convex analytic in $\mathbb{E}$ for each $\epsilon ;(|\epsilon|=$ $1)$, then $f=h+\bar{g}$ is close-to-convex harmonic in $\mathbb{E}$.

Lemma 7. If $P(z)$ and $Q(z)$ are analytic in $\mathbb{E},\left|Q^{\prime}(z)\right| \leq\left|P^{\prime}(z)\right|$ for all $z \in \mathbb{E}, Q(0)=P(0)=0$, and if $P(z)$ maps $\mathbb{E}$ onto a region which is starlike with respect to origin, then $|Q(z)| \leq|P(z)|$ for all $z \in \mathbb{E}$.

A function $\phi$ analytic in $\mathbb{E}$ is convex of order $\alpha ; 0 \leq \alpha<1$ in $\mathbb{E}$ if and only if $z \phi^{\prime}(z)$ is starlike of order $\alpha ; 0 \leq \alpha<1$ in $\mathbb{E}$ (e.g., see Duren [4]).

Lemma 8. Let $\phi$ and $\psi$ be analytic and starlike of order $1 / 2$ in $\mathbb{E}$. Then for each function $F$ analytic in $\mathbb{E}$, the convolution $(\phi * F \psi) /(\phi * \psi)$ takes only values in the convex hull of $F(\mathbb{E})$. 


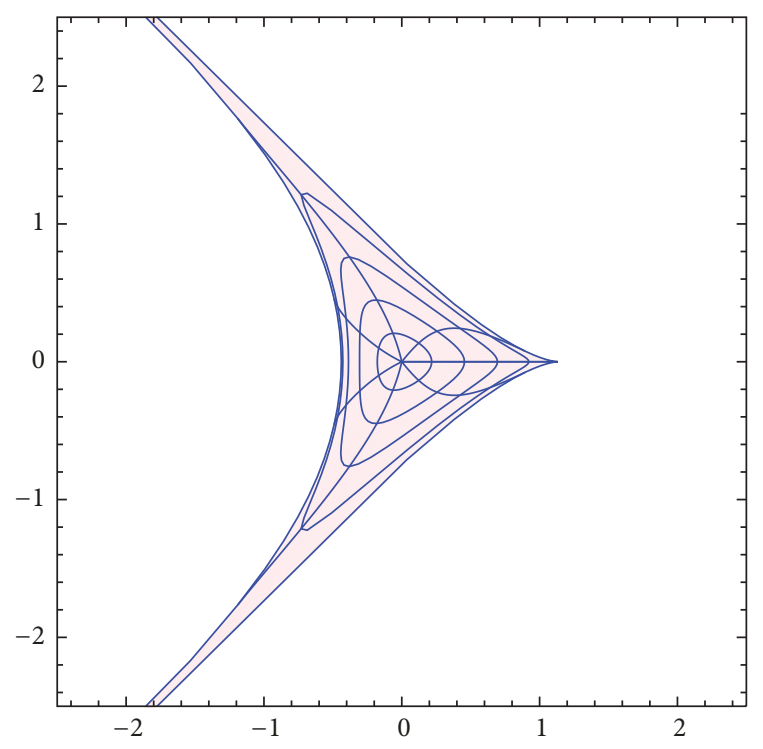

FIGURE 2: Image of $E$ under $f_{2}$.

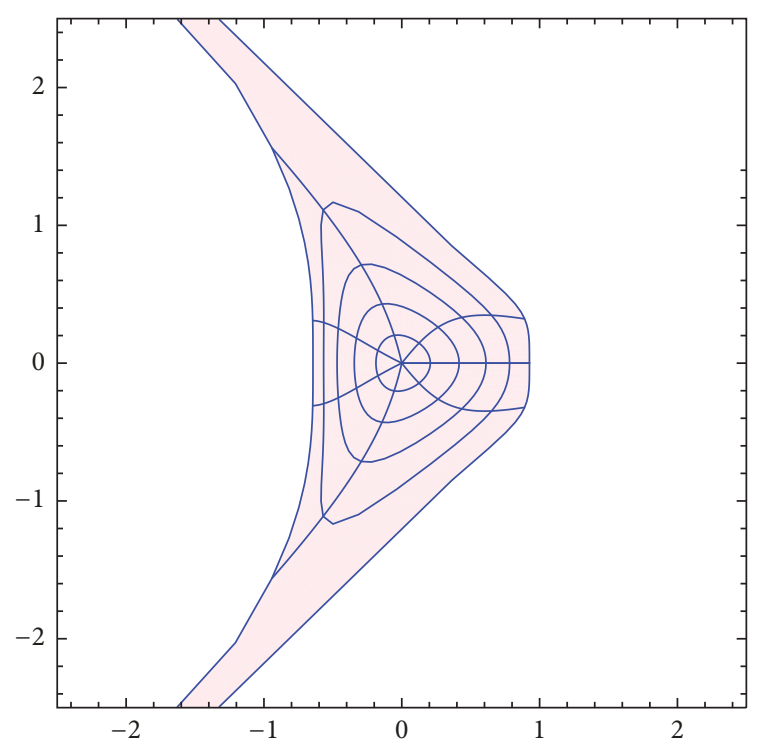

Figure 3: Image of $E$ under $f_{1} * f_{2}$.

Lemma 9. If $h$ and $g$ are analytic in $\mathbb{E}, h$ is convex in $\mathbb{E}$ and if $f=h+\bar{g}$ is locally univalent in $\mathbb{E}$, then the function $f=h+$ $\bar{g}$ is locally one-to-one, sense-preserving, and close-to-convex harmonic in $\mathbb{E}$.

Proof of Theorem 3. For the convolution function

$$
\begin{aligned}
F(z) & =h_{1}(z) * h_{2}(z)+\overline{g_{1}(z) * g_{2}(z)} \\
& =H(z)+\overline{G(z)}
\end{aligned}
$$

we note that $\left|G^{\prime}(0)\right|=0<\left|H^{\prime}(0)\right|$. To satisfy the condition of Lemma 6, we will show that $H(z)+\epsilon G(z)$ is close-to-convex analytic in $\mathbb{E}$ for each $\epsilon ;(|\epsilon|=1)$. To do so, it suffices to show that there exists a function $\phi$ analytic and convex in $\mathbb{E}$ so that

$$
\operatorname{Re} \frac{[H(z)+\epsilon G(z)]^{\prime}}{\phi^{\prime}(z)}>0 ; \quad|z|<1
$$

We observe that

$$
\begin{aligned}
{[H(z)+\epsilon G(z)]^{\prime}=} & H^{\prime}(z)+\epsilon G^{\prime}(z) \\
= & {\left[h_{1}(z) * h_{2}(z)\right]^{\prime} } \\
& +\epsilon\left[g_{1}(z) * g_{2}(z)\right]^{\prime} \\
= & {\left[\frac{1}{z} h_{1}(z) * h_{2}^{\prime}(z)\right] } \\
& +\epsilon\left[\frac{1}{z} g_{1}(z) * g_{2}^{\prime}(z)\right] \\
= & {\left[\frac{1}{z} h_{1}(z) * h_{2}^{\prime}(z)\right] } \\
& +\epsilon\left[\frac{1}{z} z^{n} h_{1}(z) * z^{n} h_{2}^{\prime}(z)\right] \\
= & {\left[\frac{1}{z} h_{1}(z) * h_{2}^{\prime}(z)\right] } \\
& +\epsilon z^{n}\left[\frac{1}{z} h_{1}(z) * h_{2}^{\prime}(z)\right] \\
= & \left(1+\epsilon z^{n}\right)\left[\frac{1}{z} h_{1}(z) * h_{2}^{\prime}(z)\right] .
\end{aligned}
$$

Now letting $\phi(z)=h_{1}(z) * h_{2}(z)$ yields

$$
\begin{aligned}
\operatorname{Re} & \frac{[H(z)+\epsilon G(z)]^{\prime}}{\phi^{\prime}(z)} \\
& =\operatorname{Re} \frac{\left(1+\epsilon z^{n}\right)\left[(1 / z) h_{1}(z) * h_{2}^{\prime}(z)\right]}{\phi^{\prime}(z)} \\
& =\operatorname{Re} \frac{\left(1+\epsilon z^{n}\right)\left[(1 / z) h_{1}(z) * h_{2}^{\prime}(z)\right]}{(1 / z) h_{1}(z) * h_{2}^{\prime}(z)} \\
& =\operatorname{Re}\left(1+\epsilon z^{n}\right)>0 .
\end{aligned}
$$

Thus by Lemma $6, F(z)=h_{1}(z) * h_{2}(z)+\overline{g_{1}(z) * g_{2}(z)}$ is locally one-to-one, sense-preserving, and close-to-convex harmonic in $\mathbb{E}$.

Proof of Theorem 4. We need to show that the convolution function

$$
\begin{aligned}
F(z) & =h_{1}(z) * h_{2}(z)+\overline{g_{1}(z) * g_{2}(z)} \\
& =H(z)+\overline{G(z)}
\end{aligned}
$$

is locally univalent and sense-preserving in $\mathbb{E}$. 
Without loss of generality, we consider only the two cases of $m=n$ and $m>n$.

Case 1. If $m=n$, then we have to show that $\left|H^{\prime}(z)\right|>\left|G^{\prime}(z)\right|$ in $\mathbb{E}$. We observe that

$$
\begin{aligned}
\left|h_{1}^{\prime}(z) * h_{2}^{\prime}(z)\right| & >\left|z^{n}\right|\left|h_{1}^{\prime}(z) * h_{2}^{\prime}(z)\right| \\
& =\left|g_{1}^{\prime}(z) * g_{2}^{\prime}(z)\right| .
\end{aligned}
$$

Inequality (10) is equivalent to

$$
\left|\left[h_{1}(z) * z h_{2}^{\prime}(z)\right]^{\prime}\right|>\left|\left[g_{1}(z) * z g_{2}^{\prime}(z)\right]^{\prime}\right|
$$

or

$$
\left|\left\{z\left[\frac{1}{z} h_{1}(z) * h_{2}^{\prime}(z)\right]\right\}^{\prime}\right|>\left|\left\{z\left[\frac{1}{z} g_{1}(z) * g_{2}^{\prime}(z)\right]\right\}^{\prime}\right|
$$

or

$$
\left|\left\{z\left[h_{1}(z) * h_{2}(z)\right]^{\prime}\right\}^{\prime}\right|>\left|\left\{z\left[g_{1}(z) * g_{2}(z)\right]^{\prime}\right\}^{\prime}\right| .
$$

Since $h_{1}(z) * h_{2}(z)$ is convex in $\mathbb{E}, z\left[h_{1}(z) * h_{2}(z)\right]^{\prime}$ is starlike in $\mathbb{E}$. Therefore, by Lemma 7 , inequality (13) yields

$$
\left|z\left[h_{1}(z) * h_{2}(z)\right]^{\prime}\right|>\left|z\left[g_{1}(z) * g_{2}(z)\right]^{\prime}\right|
$$

or

$$
\left|\left[h_{1}(z) * h_{2}(z)\right]^{\prime}\right|>\left|\left[g_{1}(z) * g_{2}(z)\right]^{\prime}\right|
$$

or

$$
\left|H^{\prime}(z)\right|>\left|G^{\prime}(z)\right|
$$

Case 2. If $m>n$, then we have

$$
\begin{aligned}
\left|h_{1}^{\prime}(z) * h_{2}^{\prime}(z)\right|> & \left|z^{n}\right|\left|h_{1}^{\prime}(z) * h_{2}^{\prime}(z)\right| \\
& =\left|z^{n} h_{1}^{\prime}(z) * z^{n} h_{2}^{\prime}(z)\right| \\
& =\left|g_{1}^{\prime}(z) * z^{n-m} g_{2}^{\prime}(z)\right| \\
& =\left|g_{1}^{\prime}(z) * g_{2}^{\prime}(z)\right| \\
& \times\left|\frac{g_{1}^{\prime}(z) * z^{n-m} g_{2}^{\prime}(z)}{g_{1}^{\prime}(z) * g_{2}^{\prime}(z)}\right| .
\end{aligned}
$$

We note that

$$
\begin{aligned}
\frac{g_{1}^{\prime}(z) * z^{n-m} g_{2}^{\prime}(z)}{g_{1}^{\prime}(z) * g_{2}^{\prime}(z)} & =\frac{z^{n} h_{1}^{\prime}(z) * z^{n-m} z^{m} h_{2}^{\prime}(z)}{z^{n} h_{1}^{\prime}(z) * z^{m} h_{2}^{\prime}(z)} \\
& =\frac{z^{n} h_{1}^{\prime}(z) * z^{n} h_{2}^{\prime}(z)}{z^{n} h_{1}^{\prime}(z) * z^{m} h_{2}^{\prime}(z)} \\
& =\frac{z^{n-1}\left[z h_{1}^{\prime}(z) * z h_{2}^{\prime}(z)\right]}{z^{n-1}\left[z h_{1}^{\prime}(z) * z^{m-n} z h_{2}^{\prime}(z)\right]} \\
& =\frac{z h_{1}^{\prime}(z) * z h_{2}^{\prime}(z)}{z h_{1}^{\prime}(z) * z^{m-n} z h_{2}^{\prime}(z)} .
\end{aligned}
$$

Letting $\phi(z)=z h_{1}^{\prime}(z), \psi(z)=z h_{2}^{\prime}(z)$, and $F(z)=z^{m-n}$ in Lemma 8 yields

$$
\left|\frac{z h_{1}^{\prime}(z) * z h_{2}^{\prime}(z)}{z h_{1}^{\prime}(z) * z^{m-n} z h_{2}^{\prime}(z)}\right|>1 .
$$

Therefore

$$
\begin{aligned}
\left|h_{1}^{\prime}(z) * h_{2}^{\prime}(z)\right|> & \left|g_{1}^{\prime}(z) * g_{2}^{\prime}(z)\right| \\
& \times\left|\frac{g_{1}^{\prime}(z) * z^{n-m} g_{2}^{\prime}(z)}{g_{1}^{\prime}(z) * g_{2}^{\prime}(z)}\right| \\
& >\left|g_{1}^{\prime}(z) * g_{2}^{\prime}(z)\right| .
\end{aligned}
$$

This is exactly inequality (10). So a similar argument following inequality (10) will lead to the conclusion that

$$
\left|H^{\prime}(z)\right|>\left|G^{\prime}(z)\right|
$$

Therefore, for either of cases $m=n$ or $m>n$,

$$
\begin{aligned}
F(z) & =h_{1}(z) * h_{2}(z)+\overline{g_{1}(z) * g_{2}(z)} \\
& =H(z)+\overline{G(z)}
\end{aligned}
$$

is locally univalent and sense-preserving in $\mathbb{E}$. Thus, by Lemma 9,

$$
F(z)=h_{1}(z) * h_{2}(z)+\overline{g_{1}(z) * g_{2}(z)}
$$

is locally one-to-one, sense-preserving, and close-to-convex harmonic in $\mathbb{E}$.

\section{Conflicts of Interest}

The authors declare that there are no conflicts of interest regarding the publication of this article.

\section{References}

[1] H. Lewy, "On the non-vanishing of the Jacobian in certain one-to-one mappings," Bulletin (New Series) of the American Mathematical Society, vol. 42, no. 10, pp. 689-692, 1936.

[2] J. Clunie and T. Sheil-Small, "Harmonic univalent functions," Annales Academiae Scientiarum Fennicae. Series A. I. Mathematica, vol. 9, pp. 3-25, 1984.

[3] P. Duren, Harmonic Mappings in the Plane, Cambridge University Press, Cambridge, UK, 2004.

[4] P. L. Duren, Univalent Functions, vol. 259, Springer Verlag, New York, NY, USA, 1983.

[5] S. Ruscheweyh and T. Sheil-Small, "Hadamard products of SCHlicht functions and the Pólya-SCHoenberg conjecture," Commentarii Mathematici Helvetici, vol. 48, pp. 119-135, 1973.

[6] O. P. Ahuja and J. M. Jahangiri, "Convolutions of harmonic functions with certain dilatations," International Journal of Mathematics and Mathematical Sciences, vol. 2017, Article ID 4015268, 4 pages, 2017.

[7] R. M. Robinson, "Univalent majorants," Transactions of the American Mathematical Society, vol. 61, pp. 1-35, 1947. 


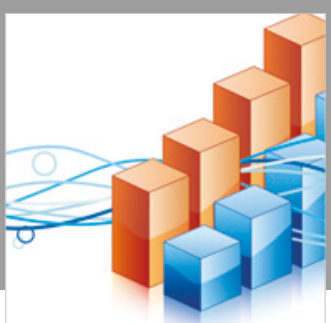

Advances in

Operations Research

\section{-n-m}
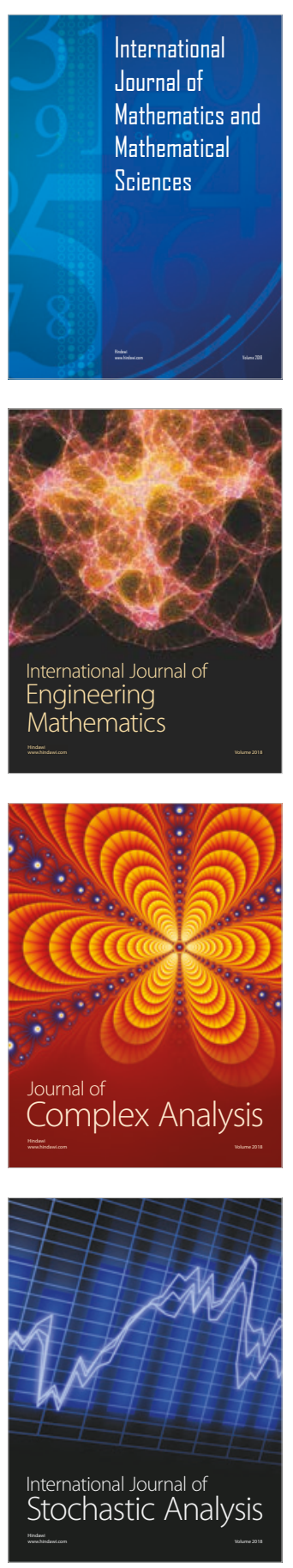
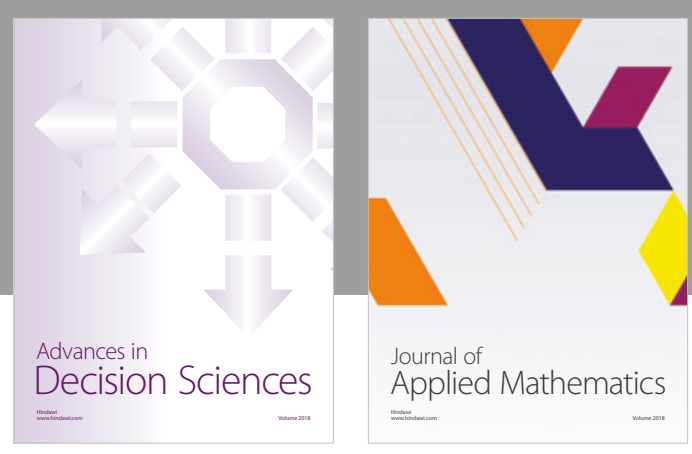

Journal of

Applied Mathematics
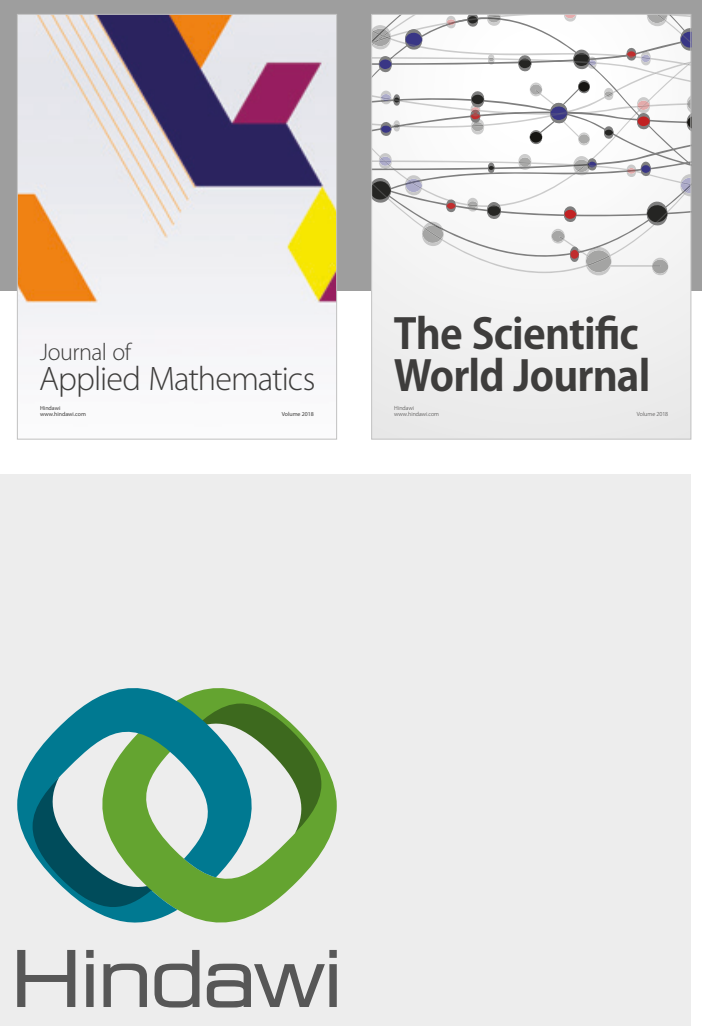

Submit your manuscripts at

www.hindawi.com

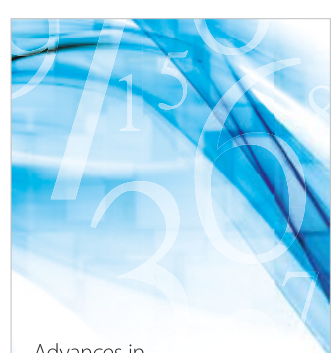

Advances in
Numerical Analysis
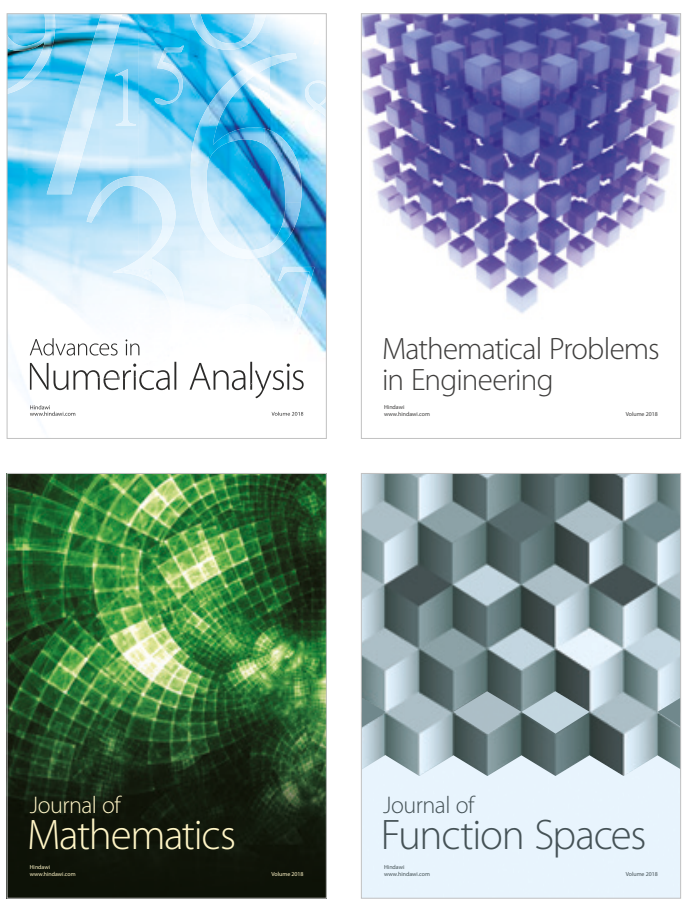

Mathematical Problems in Engineering

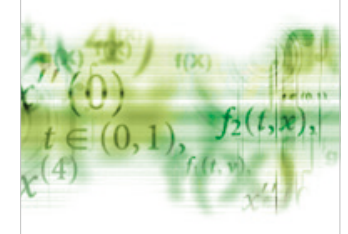

International Journal of

Differential Equations

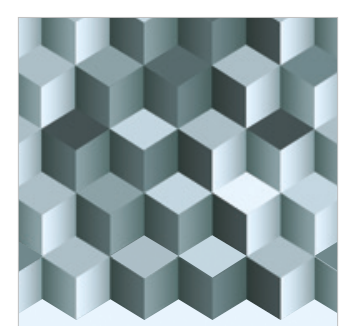

Journal of

Function Spaces

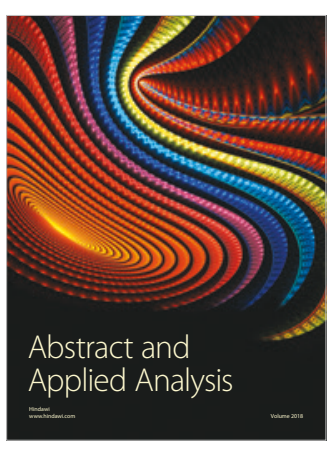

The Scientific

World Journal

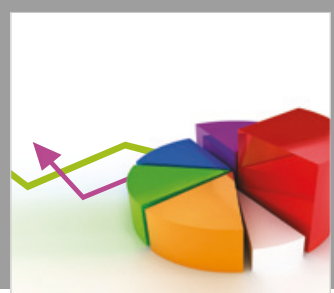

Journal of

Probability and Statistics
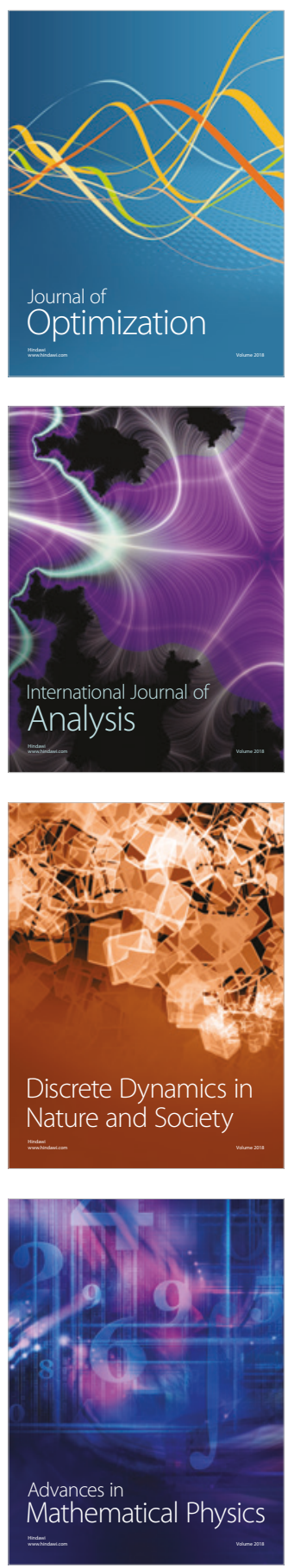